\title{
Agente intestinal bacteriano com potencial biotecnológico frente às desordens metabólicas: Uma revisão integrativa sobre a Akkermansia muciniphila
}

\author{
Intestinal bacterial agent with biotechnological potential against metabolic disorders: An \\ integrative review of Akkermansia muciniphila \\ Agente bacteriano intestinal con potencial biotecnológico contra trastornos metabólicos: Una \\ revisión integradora de Akkermansia muciniphila
}

Recebido: 22/06/2021 | Revisado: 29/06/2021 | Aceito: 05/07/2021 | Publicado: 16/07/2021

Paulo Henrique Silva

ORCID: https://orcid.org/0000-0002-2015-6497 Universidade Federal Rural de Pernambuco, Brasil E-mail: eupaulosilvabio@gmail.com

Lucas de Barros Rodrigues de Freitas ORCID: https://orcid.org/0000-0002-3127-5897 Universidade Federal Rural de Pernambuco, Brasil E-mail: lbrfreitas@hotmail.com

Sybelle Montenegro dos Santos ORCID: https://orcid.org/0000-0001-6927-4980 Universidade Federal Rural de Pernambuco, Brasil E-mail: sybellemontenegro11@gmail.com

Tarciana Lopes do Carmo

ORCID: https://orcid.org/0000-0001-9065-6393 Universidade Federal Rural de Pernambuco, Brasil E-mail: tarcianalopesbio@gmail.com

Ana Lúcia Figueiredo Porto

ORCID: https://orcid.org/0000-0001-5561-5158 Universidade Federal Rural de Pernambuco, Brasil E-mail: analuporto@yahoo.com.br

Vagne de Melo Oliveira

ORCID: https://orcid.org/0000-0003-0841-1974 Universidade Federal Rural de Pernambuco, Brasil E-mail:vagne_melo@hotmail.com

Priscilla Régia de Andrade Calaça ORCID: https://orcid.org/0000-0001-9092-6832 Universidade Federal Rural de Pernambuco, Brasil E-mail: priscilla.acalaca@ufrpe.br

Maria Taciana Cavalcanti Vieira Soares

ORCID: https://orcid.org/0000-0001-9573-6296 Universidade Federal Rural de Pernambuco, Brasil E-mail: maria.vsoares@ufrpe.br

\begin{abstract}
Resumo
Algumas doenças crônicas metabólicas são ocasionadas por disfunções alimentícias. Neste aspecto, a microbiota intestinal tem participação efetiva, direta e indiretamente, em diversos processos realizados nesse ambiente, contribuindo para a homeostase do organismo e na regularização dessas disfunções alimentícias. Recentemente, estudos publicados nos últimos anos têm demonstrado cada vez mais o papel desses microrganismos no trato gastrointestinal e como estes podem atuar como probióticos, a exemplo da bactéria Akkermansia muciniphila, a qual está interligada com vários processos bioquímicos e fisiológicos. Assim, este trabalho objetivou realizar uma revisão integrativa que compilasse informações referentes a ação da bactéria A. muciniphila contra disfunções metabólicas. Para tanto, foi realizada uma prospecção científica a partir de base de dados digitais, a saber: PubMed (https://pubmed.ncbi.nlm.nih.gov/), ScienceDirect (https://www.sciencedirect.com/) e Google Scholar (https://scholar.google.com/). Da prospecção, foram selecionados 14 artigos sobre a temática. A despeito dela, ficou evidenciada, de acordo com a literatura científica, eficiência da A. muciniphila atuando em prol do organismo de modo a prevenir e/ou tratar às desordens metabólicas decorrentes de dietas ricas, principalmente, em gorduras - fator que pode levar a quadro de obesidade e, por consequência, diabetes.
\end{abstract}

Palavras-chave: Akkermansia muciniphila; Obesidade; Microbiota intestinal; Probiótico.

\section{Abstract}

Some chronic metabolic diseases are caused by eating disorders. In this aspect, the intestinal microbiota participates effectively, directly and indirectly, in various processes carried out in this environment, contributing to the homeostasis 
of the organism and in the regularization of these nutritional disorders. Recently, studies published in recent years have increasingly demonstrated the role of these microorganisms in the gastrointestinal tract and how they can act as probiotics, such as the bacteria Akkermansia muciniphila, which is interconnected with various biochemical and physiological processes. Thus, this work aimed to carry out an integrative review to compile information regarding the action of the A. muciniphila bacteria against metabolic dysfunctions. For this purpose, a scientific survey was carried out using a digital database, namely: PubMed (https://pubmed.ncbi.nlm.nih.gov/), ScienceDirect (https://www.sciencedirect.com/) and Google Scholar (https://scholar.google.com/). From the prospection, 14 articles on the subject were selected. Despite it, according to the scientific literature, the efficiency of A. muciniphila acting on behalf of the organism was evidenced in order to prevent and/or treat metabolic disorders resulting from diets rich, mainly in fat - a factor that can lead to obesity and, consequently, diabetes.

Keywords: Akkermansia muciniphila; Obesity; Gut microbiota; Probiotic.

\section{Resumen}

Algunas enfermedades metabólicas crónicas son causadas por trastornos alimentarios. En este aspecto, la microbiota intestinal participa de forma efectiva, directa e indirecta, en varios procesos que se llevan a cabo en este medio, contribuyendo a la homeostasis del organismo y en la regularización de estos trastornos nutricionales. Recientemente, los estudios publicados en los últimos años han demostrado cada vez más el papel de estos microorganismos en el tracto gastrointestinal y cómo pueden actuar como probióticos, como la bacteria Akkermansia muciniphila, que está interconectada con diversos procesos bioquímicos y fisiológicos. Así, este trabajo tuvo como objetivo realizar una revisión integradora para recopilar información sobre la acción de la bacteria A. muciniphila frente a disfunciones metabólicas. Para ello, se realizó una encuesta científica utilizando una base de datos digital, a saber: PubMed (https://pubmed.ncbi.nlm.nih.gov/), ScienceDirect (https://www.sciencedirect.com/) y Google Académico (https://scholar.google.com/). De la prospección se seleccionaron 14 artículos sobre el tema. A pesar de ello, según la literatura científica, se evidenció la eficacia de A. muciniphila actuando en nombre del organismo para prevenir y / o tratar los trastornos metabólicos derivados de dietas ricas, principalmente en grasas, factor que puede conducir a la obesidad y, en consecuencia, diabetes.

Palabras clave: Akkermansia muciniphila; Obesidad; Microbiota intestinal; Probiótico.

\section{Introdução}

Segundo a Organização Mundial da Saúde (OMS), só em 2016, mais de 1,9 bilhão de pessoas no mundo estavam acima do peso e, com isso, a incidência de doenças associadas com à obesidade é cada vez mais frequente, principalmente entre os jovens. A obesidade é considerada uma doença crônica relacionada ao acúmulo de gordura corporal $\left(30-40 \mathrm{~kg} / \mathrm{m}^{2}\right)$ e um dos principais fatores relacionados à resistência à insulina e, consequentemente, ao desenvolvimento de diabetes tipo 2 (OMS, 2020; SBEM, 2020; Golay \& Ybarra, 2005).

A síndrome metabólica, caracterizada por circunferência abdominal elevada, costuma se desenvolver a partir dos níveis elevados de triglicerídeos, HDL e/ou pressão arterial alta, além da glicemia de jejum, podendo ser devido a outras alterações bioquímicas. Esta síndrome é sugerida como uma das principais consequências do sobrepeso, estando interligada a várias doenças, em sua maioria de caráter vascular. Somado a isto, uma dieta rica em gordura e ausência de atividade física contribuem para elevação da glicose no plasma, triglicerídeos elevados, além de alterações nas taxas de colesterol, tornando-se uma interferência na qualidade de vida, podendo levar a mortalidade (Saboya, et al., 2017; Samson, et al., 2014; Saklayen, 2018).

A diabetes mellitus (DM) é um distúrbio metabólico caracterizado por alterações no metabolismo de carboidratos, lipídeos e proteínas, relacionado com produção e/ou funcionalidade da insulina (Guthrie \& Guthrie, 2004), sendo responsável, só em 2016, por aproximadamente 1,6 milhões dos óbitos em escala mundial, segundo a OMS, estimando-se que, até 2030, pelo menos 360 milhões de pessoas desenvolvam a doença, muito devido seus hábitos sedentários (Ferreira \& Pititto, 2021; OMS, 2021). Os principais tipos de diabetes são a tipo I (insulina dependente), tipo II (insulina independente) e a gestacional (momentânea diabetes). Os índices de outras doenças crônicas em pessoas com DM são maiores, estando muitas vezes interligadas diretamente ao desenvolvimento de doenças renais e cardiovasculares, além de compor as estatísticas de prevalência de câncer (Leles, 2018). A diabetes tipo 2 está relacionada à insuficiência na produção de insulina (hiperinsulinemia) ou disfunções nas células $\beta$-pancreáticas, inviabilizando a utilização da mesma pelo organismo e constituindo um quadro clínico 
conhecido como hiperglicemia (glicose $>130 \mathrm{mg} / \mathrm{dL}$ ). Dos casos diagnosticados com DM, cerca de $90 \%$ enquadram-se no tipo 2, concentrando pessoas acima dos 40 anos e com propensão à obesidade (Fletcher, et al., 2002; Chatterjee, et al., 2017).

Estudos nos últimos anos têm demonstrado a capacidade de certos microrganismos, tanto residentes da microbiota humana, como também provenientes de suplementações (probióticos), influenciarem de forma sistêmica no organismo (Ahlawat, et al., 2021). O equilíbrio desses microrganismos no intestino é essencial, sendo benéficos para o ser humano, que pode se dar através de componentes alimentares não digeríveis (prebióticos), por estimularem a proliferação de bactérias desejáveis no cólon, favorecendo a absorção de nutrientes e o bom funcionamento dos mecanismos neuro-endócrinos (Gibson \& Roberfroid, 1995). Além disso, a colonização desses seres microscópicos no trato intestinal tem sido demonstrada como promissora quando relacionados a fatores metabólicos (Adak \& Khan, 2019). Um dos principais agentes colonizadores do trato intestinal é a bactéria Akkermansia muciniphila, degradadora de mucina (fonte de carbono, energia e nitrogênio), uma glicoproteína que protege a mucosa contra agentes infecciosos, estando presente no intestino grosso como um dos principais colonizadores, caracterizada como Gram-negativa, estritamente anaeróbia, sulfatase positiva e crescimento em pH estreito (5-6) (Devine \& Mckenzie, 1992; Derrien, et al., 2004).

As biofuncionalidades da A. muciniphila envolvem a modulação do sistema imune e a atenuação da colite ulcerativa, trazendo um novo olhar para microbiota intestinal do ponto de vista biotecnológico (Derrien, et al., 2011; Bian, et al., 2019). Desta forma, esta revisão de literatura propôs através de um modelo integrativo, concatenar os efeitos decorrentes da administração da bactéria intestinal A. muciniphila frente a doenças metabólicas acarretadas pelo consumo excessivo de gordura.

\section{Metodologia}

Este trabalho trata-se de uma revisão de literatura, a qual foi elaborada de modo integrativo (Pereira, Shitsuka, Parreira \& Shitsuka, 2018; Souza, Silva \& Carvalho, 2009). Para a busca de artigos foram utilizadas as plataformas digitais de pesquisa: PubMed (https://pubmed.ncbi.nlm.nih.gov/), ScienceDirect (https://www.sciencedirect.com/) e Google Scholar (https://scholar.google.com/), utilizando os termos (em inglês): Akkermansia muciniphila, obesity, gut microbiota, bacteria. Os fundamentos conceituais foram subdivididos nesta revisão em três tópicos: 1) Akkermansia muciniphila: alterações bioquímicas e sua ligação com o tecido adiposo; 2) Akkermansia muciniphila e expressões gênicas; e, por fim, 3) Akkermansia muciniphila e alterações quantitativas decorrentes de compostos químicos. Ainda, não havendo exclusão de artigos quanto ao período, tipo (pesquisa, revisão, comunicação rápida) e análises qualitativas e/ou quantitativas, ficando seu uso a critério dos autores.

\section{Resultados e Discussão}

A partir do levantamento de dados, perante a prospecção realizada, foi posto para a análise um total de 30 artigos, selecionando-se apenas 14 , representando $46,66 \%$ do volume total de artigos catalogados, dos quais estavam em concordância com o tema proposto. Assim sendo, descritos adiante. Sendo 50\% dos artigos utilizados para compor o tópico Akkermansia muciniphila: alterações bioquímicas e correlações com o tecido adiposo, 35,71\% para o tópico Akkermansia muciniphila e expressões gênicas e 50\% foram aproveitados para o tópico Akkermansia muciniphila: alteração decorrentes de compostos químicos.

\subsection{1) Akkermansia muciniphila: alterações bioquímicas e correlações com o tecido adiposo}

De acordo com a literatura científica, as mudanças bioquímicas decorrentes de alimentações influenciam significativamente nos reservatórios energéticos dos organismos, proporcionando melhorias e/ou disfunções metabólicas. A alimentação é um dos principais desencadeadores para as reações químicas no organismo. Ciente deste fato, a Tabela 1 apresenta os principais resultados de alterações bioquímicas referentes ao nível de glicose e triglicerídeos. Os estudos descritos correlacionaram as prováveis alterações bioquímicas em parâmetros decorrentes da influência da bactéria A. muciniphila sob as 


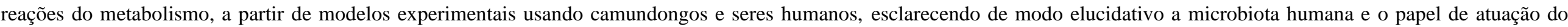
microrganismo no metabolismo.

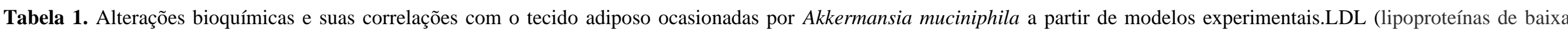
densidade) HOMAR-IR (Modelo de avaliação da homeostase); GOT (transaminase oxalacética); GPT (alanina aminotransferase); NI (Não identificado).

Experimentação

\begin{tabular}{|c|c|c|c|c|c|}
\hline Alterações bioquímicas & $\begin{array}{c}\text { Amostra } \\
\left(n^{\circ} \text { Indivíduos) }\right.\end{array}$ & $\begin{array}{l}\text { Espécie/ } \\
\text { Composto }\end{array}$ & $\begin{array}{c}\text { Concentração do } \\
\text { microrganismo }\end{array}$ & $\begin{array}{l}\text { Duração } \\
\text { (semanas) }\end{array}$ & Referências \\
\hline $\begin{array}{c}\text { Redução do ganho de massa gorda e do } \\
\text { peso corporal; } \\
\text { Redução do tecido adiposo subcutâneo, } \\
\text { epididimal e visceral; } \\
\text { Baixo índice de tecido adiposo total; } \\
\text { Aumento de atividade locomotora; } \\
\text { Aumento do conteúdo calórico fecal. }\end{array}$ & 21 camundongos & $\begin{array}{c}\text { A. } \text { muciniphila } \\
\text { pasteurizada } \\
\text { (ATTC BAA-835) }\end{array}$ & $2 \times 10^{8} \mathrm{UFC} / 180 \mu \mathrm{l} / \mathrm{dia}$ & $1-5$ & $\begin{array}{l}\text { Depommier et al. } \\
(2020)\end{array}$ \\
\hline $\begin{array}{l}\text { Menor taxa de glicose e insulina em } \\
\text { jejum; } \\
\text { Diâmetro médio dos adipócitos; } \\
\text { Melhora do colesterol total e LDL; } \\
\text { Redução no acetato sérico. }\end{array}$ & $\begin{array}{c}49 \text { participantes } \\
\text { (humanos) }\end{array}$ & A. muciniphila & NI & $0-6$ & $\begin{array}{l}\text { Dao et al. } \\
\text { (2016) }\end{array}$ \\
\hline $\begin{array}{c}\text { Diminuição da glicose em jejum; } \\
\text { Aumento no níveis séricos de insulina; } \\
\text { Redução nos níveis séricos de } \\
\text { Triglicerídeos e Colesterol total; } \\
\text { Atenuação de hiperlipidemia; } \\
\text { Redução da dimensão dos adipócitos do } \\
\text { tecido adiposo braco inguinal e tecido } \\
\text { adiposo branco epididimal; } \\
\text { Redução no diâmetro dos adipócitos } \\
\text { inguinal. }\end{array}$ & 60 camundongos & $\begin{array}{c}\text { Akkermansia } \\
\text { muciniphila } \\
\text { (Amuc_GP01e } \\
\text { Amuc_GP25) }\end{array}$ & $5 \times 10^{9} \mathrm{UFC} / \mathrm{mL} / \mathrm{dia}$ & $0-16$ & Deng et al. (2020) \\
\hline
\end{tabular}


Redução do ganho de peso, ingestão calórica, peso da gordura mesentérica, gordura total, gordura subcutânea,

$$
\text { gordura epididimal; }
$$

Redução de eficiência energética;

Redução do diâmetro médio dos

$$
\text { adipócitos; }
$$

Redução do colesterol total;

Redução dos níveis séricos de glicose, insulina, HOMA-IR;

Redução sérica de GOT, GPT

Redução de massa gorda;

Aumento de massa magra;

Tolerância à glicose

Redução no acúmulo de lipídios no

fígado e músculos.

Aumento nos níveis intestinais de endocanabinóides;

Controle do armazenamento de massa de gordura;

Controle da homeostase da glicose

Reverteu a resistência à insulina;

Regulação da barreira intestinal;

Redução da endotoxemia metabólica.

Diminuição do peso corporal e gordura

$$
\text { retroperitoneal; }
$$

Diminuição do peso dos rins.

\section{9 camundongos}

\section{A. muciniphila \\ pasteurizada \\ EB-AMDK 10, EB-}

AMDK 19, and EB-

AMDK 27)

\section{$20 / 40$}

Camundongos

C57BL/6

NI

camundongos

C57BL/6

A. muciniphila

$\mathrm{Muc}^{\mathrm{T}}$ (ATTC BAA-

$835)$

$2,10^{8} \mathrm{UFC} / 0,2 \mathrm{~mL} / \mathrm{dia}$

$0-4$

Everard et al.

(2013)

Zhao et al

(2017)

$$
\text { BAA-835) }
$$

2 x $10^{8} \mathrm{UFC} / 200 \mu \mathrm{l} / \mathrm{dia}$

4 grupos $(\mathrm{n}=8-12$

grupo)

A. muciniphila

$2 \times 10^{8} \mathrm{UFC} / 150 \mu \mathrm{l} / \mathrm{dia}$

0-4

Lawenius et al

(2020)

Camundongos

C57BL / 6

Fonte: Autores. 
De acordo com os autores reportados na Tabela 1, pode-se observar que ao analisar administração da A. muciniphila em seres humanos, como propósito de intervenção, Dao et al. (2016) obtiveram resultados significativos, verificando-se uma diminuição dos níveis de triglicerídeos em jejum $\{[1,2(0,9-1,7) \mathrm{mmol} / \mathrm{L}$ para $1,0(0,8-1,2)] \mathrm{mmol} / \mathrm{L}\}$, contribuindo para a redução de gordura, tendo em vista que os triglicerídeos são as principais responsáveis por doenças relacionadas à aterosclerose. Ainda segundo os autores, a bactéria promoveu a diminuição do tamanho médio dos adipócitos, menor mediana de gordura ginóide e menor mediana de gordura andróide, o que levou as inflamações no tecido adiposo a diminuir. Vale ressaltar que, a correlação dos níveis de A. muciniphila, mesmo com a intervenção dietética, o grupo com altos níveis de A. muciniphila apresentou maiores benefícios (Dao, et al., 2016).

Depommier et al. (2020), em trabalho referente a perda de gordura correlacionada com A. muciniphila através de experimentação realizado em câmaras metabólicas, A. muciniphila pasteurizada, promoveram em camundongos obesos a preferência pela utilização de lipídeos em contraste com magros, os quais preferiram o uso da glicose para realização de atividades locomotoras. Ainda segundo os autores, foram observados uma dificuldade de ganho de massa gorda em apenas duas semanas, evidenciando a capacidade deste microrganismo em evitar o ganho de peso corporal. Everard et al. (2013) reportaram a capacidade da A. muciniphila em contornar os níveis elevados de glicose em jejum em decorrência de dieta hipercalórica. Como observado pelos autores, uma das competências da bactéria está ligada à atenuação de inflamações ocorridas no tecido adiposo em virtude do aumento de gordura. Entre esses fatores benéficos, está o aumento nos níveis de endocanabinóides estimula a secreção de peptídeos intestinais, tais como GLP-1 (Glucagon 1 tipo), sendo este estimulante da secreção de insulina, supressor de glucagon e, além disso, retarda o esvaziamento gástrico. Assim, esses neurotransmissores contribuem para a perda de gordura, em decorrência da redução no consumo de alimentos.

Ao analisar o potencial de três cepas bacterianas pasteurizada de A. muciniphila (EB-AMDK-10, EB-AMDK-19 e EBAMDK-27) administrada em uma dosagem de $1 \times 10^{8}$ UFC/animal em camundongos, isoladas de amostras fecais humana, Yang et al. (2020) observaram a redução nos níveis de glicose mesmo com a administração de dietas hipercalóricas. Ainda sendo observação dos autores, as cepas foram capazes de contornar mudanças negativas no tecido adiposo, apresentando-as com efeitos significativos na diminuição de ganho de peso, diminuição de gordura mesentérica, gordura epididimal e tamanho médio dos adipócitos (Yang, et al., 2020).

A perda de peso utilizando a bactéria como probiótico é uma prática válida e que tem sido constatada através de experimentação (Everard, et al., 2013). Ao administrar duas cepas de A. muciniphila (Amuc_GP01 e Amuc_GP25) por gavagem em camundongos tratados com dieta hipercalórica, Deng et al. (2020) constataram a diminuição da glicose em jejum, diminuição sérica de triglicerídeos e colesterol total, além de suas influências no tecido adiposo branco inguinal e epididimal. Estes resultados vão de encontro com os descritos por Zhao et al. (2017) que utilizaram uma dosagem de $1 \times 10^{8}$ UFC/200 $\mu 1$ da A. muciniphila, durante um período de cinco semanas e observaram uma diminuição do ganho de peso corporal, massa gorda e aumento da massa magra; teoria corroborada por Lawenius et al. (2020) ao utilizarem A. muciniphila pasteurizada e observarem a minimização da gorduras retroperitoneal em camundongos, sinalizando a potencialidade da bactéria como agente probiótico a partir de suas diferentes cepas, em atuar na redução de gordura nos diferentes tecidos corporais.

Indubitavelmente, as disposições das gorduras, o ganho de massa magra e a homeostase nos níveis séricos de glicose são exemplos das alterações bioquímicas causadas pela A. muciniphila, podendo contribuir para o bem-estar dos indivíduos, na resistência a ganho de peso como também na prevenção da diabetes.

\subsection{2) Akkermansia muciniphila e expressões gênicas}

As relações indiretas desse microrganismo junto às expressões gênicas estão cada vez mais em evidência na literatura científica. A relação da bactéria com as diferentes expressões gênicas é descrita na tabela 2, observando-se a normalização e o aumento/diminuição em diferentes partes dos tecidos corporais. 


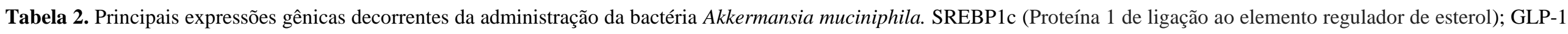

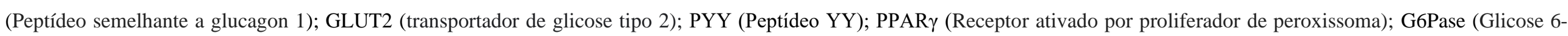

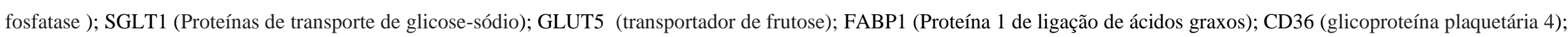

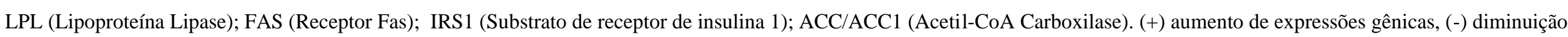
de expressão gênicas, (+/-) regulação de expressão gênicas.

\begin{tabular}{|c|c|c|c|c|c|c|c|c|c|c|c|c|c|c|c|c|c|}
\hline & & \multicolumn{15}{|c|}{ Expressões gênicas } & \multirow[b]{2}{*}{ Referências } \\
\hline $\begin{array}{l}\text { Bactéria/ } \\
\text { Espécie }\end{array}$ & Dosagem & $\begin{array}{l}\text { SREBP } \\
1 \mathrm{c}\end{array}$ & GLP-1 & GLUT2 & PYY & $\operatorname{PPAR} \gamma$ & G6Pase & SGLT1 & GLUT5 & FABP1 & CD36 & LPL & FAS & IRS1 & ACC & ACC1 & \\
\hline $\begin{array}{l}\text { ATTC BAA- } \\
\quad 835\end{array}$ & $\begin{array}{c}2 \times 10^{8} \mathrm{UFC} / 180 \\
\mu \mathrm{l} / \mathrm{dia}\end{array}$ & & & - & & & & - & - & + & + & & & & & & $\begin{array}{c}\text { Depommier et al. } \\
\text { (2020) }\end{array}$ \\
\hline EB-AMDK 10 & $\begin{array}{c}1 \times 10^{8} \text { UFC/ } 6 \\
\text { vezes por } \\
\text { semana }\end{array}$ & - & $+/-$ & & + & - & - & & & & & $+/-$ & - & + & & - & $\begin{array}{l}\text { Yang et al. } \\
\text { (2020) }\end{array}$ \\
\hline EB-AMDK 19 & $\begin{array}{c}1 \times 10^{8} \mathrm{UFC} / 6 \\
\text { vezes por } \\
\text { semana }\end{array}$ & - & & + & + & - & - & & & & - & $+/-$ & - & + & & - & $\begin{array}{l}\text { Yang et al. } \\
\text { (2020) }\end{array}$ \\
\hline EB-AMDK 27 & $\begin{array}{c}1 \times 10^{8} \mathrm{UFC} / 6 \\
\text { vezes por } \\
\text { semana }\end{array}$ & - & $+/-$ & + & + & - & - & & & & - & $+/-$ & - & + & & - & $\begin{array}{l}\text { Yang et al. } \\
(2020)\end{array}$ \\
\hline BAA-835 & $\begin{array}{c}10^{8} \text { a } 10^{9} \mathrm{UFC} / \\
\mathrm{ml} / \mathrm{dia}\end{array}$ & - & & & & & & & & & & & & & & & $\begin{array}{l}\text { Kim et al. } \\
(2020)\end{array}$ \\
\hline BAA-835 & $10^{8} \mathrm{UFC} / \mathrm{dia}$ & - & & & & & & & & & & - & & & & & $\begin{array}{l}\text { Sheng et al. } \\
\text { (2018) }\end{array}$ \\
\hline $\begin{array}{l}\text { ATCC BAA- } \\
\quad 835\end{array}$ & $\begin{array}{c}2 \times 10^{8} \text { UFC / } \\
200 \mu \mathrm{l} / \mathrm{dia}\end{array}$ & - & & & & & & & & & & & & & - & & $\begin{array}{l}\text { Zhao et al. } \\
\text { (2017) }\end{array}$ \\
\hline
\end{tabular}

Fonte: Autores. 
De acordo com os dados ilustrados na Tabela 2, é possível observar que quando da experimentação com camundongos, houve uma diminuição das expressões gênicas de LPL (Lipoproteína lipase) e SREBP 1c (Proteína 1 de ligação ao elemento regulador de esterol), envolvidas na adipogênese e derivadas de adipócitos em tecido adiposo branco e também na lipogênese (Sheng, et al., 2018; Zhao, et al., 2017). Também pode ser observado em Yang et al. (2020) a redução das expressões gênicas de SREBP 1c no tecido adiposo quando utilizaram três cepas de A. muciniphila pasteurizada em oposição à desordens metabólicas. Além disso, os autores também relataram uma diminuição e/ou regulação de expressões gênicas de GLUT2 (Transportador de glicose 2), GLP 1 (1 tipo de glucagon), como também de PYY (Peptídeo YY), sendo estes dois últimos essenciais na regulação da fome e da saciedade.

A utilização da bactéria estudada também reduziu significativamente a expressão de SREBP no fígado, sendo neste caso a administração média de $10^{8}$ a $10^{9} \mathrm{UFC} /$ dia. Esse efeito culminou na redução de síntese de triglicérides no órgão e, consequentemente, na minimização de danos hepáticos (Kim, et al., 2020). O emprego de A. muciniphila pasteurizada (ATTC BAA-835) durante 5 semanas com uma dosagem de $2 \times 10^{8} \mathrm{UFC} /$ administrada $180 \mu \mathrm{l} / \mathrm{dia}$ em camundongos, proporcionou o aumento de CD36 (glicoproteína plaquetária 4) e FABP1 (Proteína 1 de ligação a ácidos graxos), ambos estando ligados diretamente com melhoras no perfil lipídico. Além disso, conforme demonstrado, houve expressões de GLUT2 (Depommier, et al., 2020). As expressões gênicas demonstradas nos trabalhos citados na tabela 2 reforçam a eficiência da A. muciniphila em amenizar sistemicamente as desordens metabólicas acarretadas pelo excesso de gordura, diminuindo as chances de desenvolver doenças crônicas ligadas à as expressões gênicas.

\subsection{3) Akkermansia muciniphila: alteração decorrentes de compostos químicos}

Os estudos explanados nesta revisão demonstram a capacidade de determinadas substâncias químicas, presentes em alimentos ou suplementadas de forma intencional em interferirem no crescimento da A. muciniphila e, assim, somados aos prováveis efeitos que ela apresenta, podendo melhorar ou não os principais parâmetros metabólicos. Everard et al. (2013) evidenciaram a importância da suplementação com prebióticos para aumentar os níveis da bactéria avaliada, sendo esse benefício já relacionado a microbiota intestinal. Em seus estudos, ao analisarem administração de oligofrutose em camundongos, os níveis da A. muciniphila foram regularizados, bem como a diminuição no ganho de massa gorda, sendo sugestionado pelos autores como uma das consequências do aumento dessa bactéria no intestino. Ainda, observam-se estudos relacionados aos polifenóis, compostos químicos encontrados principalmente em frutas e verduras, com o aumento da A. muciniphila. A utilização, por exemplo, de polifenóis de uva concord e Galato de epigalocatequina, outro tipo de polifenol, encontrado principalmente em ervas verdes, enriqueceram significativamente os níveis de A. muciniphila no intestino de camundongos (Roopchand, et al., 2015; Sheng, et al., 2018)

Capsaicina, presente na pimenta e no pimentão, também promoveu o crescimento desse microrganismo na microbiota intestinal, saindo da concentração de 0,03 $\pm 0,04 \%$ para $0,12 \pm 0,07 \%$ (Shen et al., 2017). No trabalho de De La Cuesta-Zuluaga et al. (2017), a utilização de metformina, composto utilizado por pacientes com diabete tipo 1, aumentou consideravelmente os níveis de A. muciniphila no intestino e, em decorrência desses acontecimentos, a mediação na redução de glicose poderia ser concretizada por essa bactéria. Outro fármaco, a vancomicina, um antibiótico, elevou a concentração também de A.muciniphila (Hansen, et al., 2012). Em contraste, o consumo de alimentos ricos em gorduras levaram a uma diminuição da quantidade de $A$. muciniphila em camundongos, chegando a ser dez mil vezes menor que a do grupo controle, o qual apresentou uma diminuição de cem vezes (Schneeberger, et al., 2015). Com esses resultados, é possível inferir que existe uma influência direta da alimentação na composição da microbiota intestinal e, por consequência, em seus efeitos disbióticos e metabólicos. 


\section{Conclusão}

A partir das prospecções realizadas em artigos científicos e em plataformas digitais, este trabalho abordou de modo circunstancial e pontual as principais informações inerentes à utilização da bactéria Akkermansia muciniphila como agente probiótico, junto às desordens metabólicas, especialmente as relacionadas a obesidade e a diabetes. A utilização da A. mисіniphila, a partir das experimentações realizadas e descritas na literatura, demonstrou eficiência em contornar mudanças metabólicas provocadas pelo consumo excessivo e acúmulo de gordura. Diante desses efeitos, é notória a importância deste microrganismo para a microbiota humana. Assim, esperamos que com este trabalho de revisão, estudantes, pesquisadores e profissionais da área de microbiologia e biotecnologia possam utilizar os dados como suporte para auxiliar em futuras investidas no campo das enfermidades crônicas relacionadas com distúrbios bioquímicos, especialmente os crônicos, tornando-se uma ferramenta colaborativa para o entendimento nutracêutico da A. muciniphila. Além da possibilidade de realização de experimentos com esta bactéria em outros contextos metabólicos não avaliados nessa revisão.

\section{Referências}

Ahlawat, S., Asha, \& Sharma, K. K. (2021). Gut-organ axis: a microbial outreach and networking. Letters in applied microbiology, 72(6), 636-668. https://doi.org/10.1111/lam.13333.

Adak, A., \& Khan, M. R. (2019). An insight into gut microbiota and its functionalities. Cellular and molecular life sciences: CMLS, 76(3), 473-493. https://doi.org/10.1007/s00018-018-2943-4.

Bian, X., Wu, W., Yang, L., Lv, L., Wang, Q., Li, Y., Ye, J., Fang, D., Wu, J., Jiang, X., Shi, D., \& Li, L. (2019). Administration of Akkermansia muciniphila Ameliorates Dextran Sulfate Sodium-Induced Ulcerative Colitis in Mice. Frontiers in microbiology, 10, 2259. https://doi.org/10.3389/fmicb.2019.02259

Chatterjee, S., Khunti, K., \& Davies, M. J. (2017). Type 2 diabetes. Lancet, 389(10085), 2239-2251. https://doi.org/10.1016/S0140-6736(17)30058-2

Devine, P. L., \& McKenzie, I. F. (1992). Mucins: structure, function, and associations with malignancy. BioEssays : news and reviews in molecular, cellular and developmental biology, 14(9), 619-625. https://doi.org/10.1002/bies.950140909

Derrien, M., Vaughan, E. E., Plugge, C. M., \& de Vos, W. M. (2004). Akkermansia muciniphila gen. nov., sp. nov., a human intestinal mucin-degrading bacterium. International journal of systematic and evolutionary microbiology, 54(5), 1469-1476. https://doi.org/10.1099/ijs.0.02873-0

Derrien, M., Van Baarlen, P., Hooiveld, G., Norin, E., Müller, M., \& de Vos, W. M. (2011). Modulation of Mucosal Immune Response, Tolerance, and Proliferation in Mice Colonized by the Mucin-Degrader Akkermansia muciniphila. Frontiers in microbiology, 2, 166. https://doi.org/10.3389/fmicb.2011.00166

Depommier, C., Van Hul, M., Everard, A., Delzenne, N. M., De Vos, W. M., \& Cani, P. D. (2020). Pasteurized Akkermansia muciniphila increases whole-body energy expenditure and fecal energy excretion in diet-induced obese mice. Gut microbes, 11(5), 1231-1245. https://doi.org/10.1080/19490976.2020.1737307

Dao, M. C., Everard, A., Aron-Wisnewsky, J., Sokolovska, N., Prifti, E., Verger, E. O., Kayser, B. D., Levenez, F., Chilloux, J., Hoyles, L., MICRO-Obes Consortium, Dumas, M. E., Rizkalla, S. W., Doré, J., Cani, P. D., \& Clément, K. (2016). Akkermansia muciniphila and improved metabolic health during a dietary intervention in obesity: relationship with gut microbiome richness and ecology. Gut, 65(3), 426-436. https://doi.org/10.1136/gutjnl-2014-308778

Deng, L., Ou, Z., Huang, D., Li, C., Lu, Z., Liu, W., Wu, F., Nong, C., Gao, J., \& Peng, Y. (2020). Diverse effects of different Akkermansia muciniphila genotypes on Brown adipose tissue inflammation and whitening in a high-fat-diet murine model. Microbial pathogenesis, 147. https://doi.org/10.1016/j.micpath.2020.104353

De la Cuesta-Zuluaga, J., Mueller, N. T., Corrales-Agudelo, V., Velásquez-Mejía, E. P., Carmona, J. A., Abad, J. M., \& Escobar, J. S. (2017). Metformin Is Associated With Higher Relative Abundance of Mucin-Degrading Akkermansia muciniphila and Several Short-Chain Fatty Acid-Producing Microbiota in the Gut. Diabetes care, 40(1), 54-62. https://doi.org/10.2337/dc16-1324

Everard, A., Belzer, C., Geurts, L., Ouwerkerk, J. P., Druart, C., Bindels, L. B., Guiot, Y., Derrien, M., Muccioli, G. G., Delzenne, N. M., de Vos, W. M., \& Cani, P. D. (2013). Cross-talk between Akkermansia muciniphila and intestinal epithelium controls diet-induced obesity. Proceedings of the National Academy of Sciences of the United States of America, 110(22), 9066-9071. https://doi.org/10.1073/pnas.1219451110

Ferreira, S. R. G \& Pititto, B. A. (2021). Aspectos Epidemiológicos do Diabetes Mellitus e seu Impacto no Indivíduo e na Sociedade. https://ebook.diabetes.org.br/component/k2/item/73-capitulo-1-aspectos-epidemiologicos-do-diabetes-mellitus-e-seu-impacto-no-individuo-e-na-sociedade

Fletcher, B., Gulanick, M., \& Lamendola, C. (2002). Risk factors for type 2 diabetes mellitus. The Journal of cardiovascular nursing, 16(2), 17-23. https://doi.org/10.1097/00005082-200201000-00003

Golay, A., \& Ybarra, J. (2005). Link between obesity and type 2 diabetes. Best practice \& research. Clinical endocrinology \& metabolism, 19(4), 649-663. https://doi.org/10.1016/j.beem.2005.07.010

Guthrie, R. A., \& Guthrie, D. W. (2004). Pathophysiology of diabetes mellitus. Critical care nursing quarterly, 27(2), 113-125. https://doi.org/10.1097/00002727-200404000-00003 
Gibson, G. R., \& Roberfroid, M. B. (1995). Dietary modulation of the human colonic microbiota: introducing the concept of prebiotics. The Journal of nutrition, 125(6), 1401-1412. https://doi.org/10.1093/jn/125.6.1401

Hansen, C. H., Krych, L., Nielsen, D. S., Vogensen, F. K., Hansen, L. H., Sørensen, S. J., Buschard, K., \& Hansen, A. K. (2012). Early life treatment with vancomycin propagates Akkermansia muciniphila and reduces diabetes incidence in the NOD mouse. Diabetologia, 55(8), 2285-2294. https://doi.org/10.1007/s00125-012-2564-7

Kim, S., Lee, Y., Kim, Y., Seo, Y., Lee, H., Ha, J., Lee, J., Choi, Y., Oh, H., \& Yoon, Y. (2020). Akkermansia muciniphila Prevents Fatty Liver Disease, Decreases Serum Triglycerides, and Maintains Gut Homeostasis. Applied and environmental microbiology, 86(7), e03004-19. https://doi.org/10.1128/AEM.03004-19

Leles, M. B. L. (2018). Influência da Diabetes Mellitus nas doenças crônicas e agudas. Pebmed. https://pebmed.com.br/influencia-da-diabetes-mellitus-nasdoencas-cronicas-e-agudas/

Lawenius, L., Scheffler, J. M., Gustafsson, K. L., Henning, P., Nilsson, K. H., Colldén, H., Islander, U., Plovier, H., Cani, P. D., de Vos, W. M., Ohlsson, C., \& Sjögren, K. (2020). Pasteurized Akkermansia muciniphila protects from fat mass gain but not from bone loss. American journal of physiology, Endocrinology and metabolism, 318(4), E480-E491. https://doi.org/10.1152/ajpendo.00425.2019

Pereira, A. S., Shitsuka, D. M., Parreira, F. J., \& Shitsuka, R. (2018). Metodologia da pesquisa científica. UFSM.

Roopchand, D. E., Carmody, R. N., Kuhn, P., Moskal, K., Rojas-Silva, P., Turnbaugh, P. J., \& Raskin, I. (2015). Dietary Polyphenols Promote Growth of the Gut Bacterium Akkermansia muciniphila and Attenuate High-Fat Diet-Induced Metabolic Syndrome. Diabetes, 64(8), 2847-2858. https://doi.org/10.2337/db141916

Saboya, P. P., Bodanese, L. C., Zimmermann, P. R., Gustavo, A. D., Macagnan, F. E., Feoli, A. P., \& Oliveira, M. D. (2017). Lifestyle Intervention on Metabolic Syndrome and its Impact on Quality of Life: A Randomized Controlled Trial. Arquivos brasileiros de cardiologia, 108(1), 60-69. https://doi.org/10.5935/abc.20160186

Samson, S. L., \& Garber, A. J. (2014). Metabolic syndrome. Endocrinology and metabolism clinics of North America, 43(1), 1-23. https://doi.org/10.1016/j.ecl.2013.09.009

Saklayen, M. G. (2018). The Global Epidemic of the Metabolic Syndrome. Current hypertension reports, 20(2), 12. https://doi.org/10.1007/s11906-018-0812-z

Sociedade Brasileira de Endocrinologia e Metabologia. (2020). A Obesidade é uma doença crônica. https://www.sbemsp.org.br/imprensa/releases/736-aobesidade-e-uma-doenca

Souza, M. T., Silva, M. D., Carvalho, R. (2010). Revisão integrativa: o que é e como fazer? Einstein, 8(1). https://doi.org/10.1590/S1679-45082010RW1134

Shen, W., Shen, M., Zhao, X., Zhu, H., Yang, Y., Lu, S., Tan, Y., Li, G., Li, M., Wang, J., Hu, F., \& Le, S. (2017). Anti-obesity Effect of Capsaicin in Mice Fed with High-Fat Diet Is Associated with an Increase in Population of the Gut Bacterium Akkermansia muciniphila. Frontiers in microbiology, 8, 272. https://doi.org/10.3389/fmicb.2017.00272

Schneeberger, M., Everard, A., Gómez-Valadés, A. G., Matamoros, S., Ramírez, S., Delzenne, N. M., Gomis, R., Claret, M., \& Cani, P. D. (2015). Akkermansia muciniphila inversely correlates with the onset of inflammation, altered adipose tissue metabolism and metabolic disorders during obesity in mice. Scientific reports, 5, 16643. https://doi.org/10.1038/srep16643

Sheng, L., Jena, P. K., Liu, H. X., Hu, Y., Nagar, N., Bronner, D. N., Settles, M. L., Bäumler, A. J., \& Wan, Y. Y. (2018). Obesity treatment by epigallocatechin3-gallate-regulated bile acid signaling and its enriched Akkermansia muciniphila. FASEB journal: official publication of the Federation of American Societies for Experimental Biology, 32(12), fj201800370R. https://doi.org/10.1096/fj.201800370R

World Health organization. (2021). Diabetes. https://www.who.int/es/news-room/fact-sheets/detail/diabetes.

World Health Organization. (2020). Obesity and overweight. https://www.who.int/news-room/fact-sheets/detail/obesity-and-overweight.

Yang, M., Bose, S., Lim, S., Seo, J., Shin, J., Lee, D., Chung, W. H., Song, E. J., Nam, Y. D., \& Kim, H. (2020). Beneficial Effects of Newly Isolated Akkermansia muciniphila Strains from the Human Gut on Obesity and Metabolic Dysregulation. Microorganisms, 8(9), 1413. https://doi.org/10.3390/microorganisms8091413

Zhao, S., Liu, W., Wang, J., Shi, J., Sun, Y., Wang, W., Ning, G., Liu, R., \& Hong, J. (2017). Akkermansia muciniphila improves metabolic profiles by reducing inflammation in chow diet-fed mice. Journal of molecular endocrinology, 58(1), 1-14. https://doi.org/10.1530/JME-16-0054 